\title{
Estudio de la gestión social y productiva local de la actividad agrícola en la comunidad de Yanallpa, Requena, Perú
}

\section{Study of social management and productive local of agricultural activity in the community of Yanallpa, Requena, Perú}

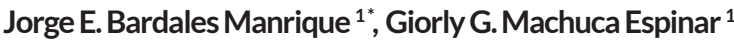 \\ ${ }^{1}$ Universidad Nacional de la Amazonía Peruana
}

\section{RESUMEN}

Los objetivos fueron, describir los saberes, prácticas de cultivo, composición florística y formas de uso del huerto-chacra en la comunidad de Yanallpa a fin de contribuir con su mejor comprensión. Se registraron un total de 39 especies; identificando 2 tipos y 4 subtipos de cultivo; la diversidad de especies encontradas fue hasta 3,39 para el índice de Margalef y 1,54 para el índice de Shannon. La similaridad en pares de huerto-chacras tuvo un máximo de $51 \%$ y un mínimo de $5 \%$ de especies compartidas. Las principales categorías de uso reportadas fueron alimento, medicina, leña, material de construcción y uso doméstico. La orientación de la producción fue mayormente mixta; existe una asociación de plantas reflejada mayormente en diversidad de especies que, en cantidad de individuos, con altos índices de diversidad determinados principalmente por las necesidades e intereses de sus propietarios. Concluyendo, se observa que el cuidado del huerto-chacra está muy relacionado con la familia y la importancia y afecto que da al cultivo de sus plantas, ya que los miembros adultos a través de sus conocimientos ancestrales y locales han logrado un manejo adecuado de las especies, por ello la emocionalidad y la ritualidad están presentes, a tal punto que desconocer la cosmovisión local y más aún, no comprenderla a pesar de conocerla; puede poner en riesgo el éxito de los proyectos de desarrollo en comunidades como Yanallpa.

Palabras clave: Gestión social, gestión productiva, índices diversidad biológica, huertos chachas.

\section{ABSTRACT}

The objectives were to describe the knowledge, cultivation practices, floristic composition and ways of using the garden farm in the community of Yanallpa in order to contribute to its better understanding. A total of 39 species were recorded; identifying 2 types and 4 culture subtypes; the diversity of species found was up to 3,39 for the Margalef index and 1,54 for the Shannon index. The similarity in pairs of gardenfarm had a maximum of $51 \%$ and a minimum of $5 \%$ of shared species. The main categories of use reported were food, medicine, firewood, construction material and domestic use. The orientation of the production was mostly mixed; there is an association of plants reflected mostly in diversity of species that, in number of individuals, with high diversity indices determined mainly by the needs and interests of their owners. In conclusion, it is observed that the care of the garden farm is very related to the family and the importance and affection that it gives to the cultivation of its plants, since the adult members through their ancestral and local knowledge have managed an adequate management of the species, for that reason the emotionality and the rituality are present, to such a point that to ignore the local cosmovision and even more, not to understand it in spite of knowing it; can put at risk the success of development projects in communities like Yanallpa.

Keywords: Social management, productive management, indices biological diversity, orchards chachas.

Historial del artículo:

Recibido, 02 de marzo 2016; aceptado, 10 de mayo de 2016; disponible en línea, 15 de julio de 2016

* Docente de la Universidad Nacional de la Amazonía Peruana

Correo: jobama52@hotmail.com 


\section{INTRODUCCIÓN}

El río Ucayali, específicamente la cuenca baja, es una zona de características biofísicas correspondiente a terrenos aluviales con alta fertilidad de suelos (Encarnación, 1993), pero afectada con periodos de inundación total, que en ocasiones pueden ser muy prolongados generando problemas en la economía del poblador ribereño, esto generalmente debido a la demora en la aparición de los suelos de barriales en la cual desarrollan una parte importante de su actividad agrícola.

En este contexto, dentro de la cuenca baja del río Ucayali se ubica la comunidad de Yanallpa, pequeño poblado de unos 487 habitantes aproximadamente, asentada en terrenos inundables, donde existe la práctica generalizada de una forma de agricultura denominada y descrita por De Jong (1995) como el "huerto-chacra" o "jardín boscoso" (forest garden) que muestra patrones de cultivo y composición de especies altamente variables, más que cualquier otro tipo de agricultura descrito anteriormente; como consecuencia de los procesos de selección, domesticación y adaptación de especies nativas.

Los agricultores de la comunidad de Yanallpa vienen desarrollando durante muchos años estos sistemas agrícolas productivos con características particulares, que los ha diferenciado de otras comunidades. Entre las principales se mencionan la forma de uso del suelo, la organicidad sociocultural para el trabajo agrícola, una mayor diversidad de cultivos dentro de la parcela, uso de coberturas en los cultivos, el reciclaje y uso de residuos orgánicos y un manejo limpio libre de productos químicos; todo ello en base a sus saberes agroecológicos que son definidos como una constelación de conocimientos, técnicas y prácticas dispersas que responden a las condiciones ecológicas, económicas, técnicas y culturales de cada geografía y cada población. Estos saberes se forjan en la interface entre las cosmovisiones, teorías y prácticas (Leff, 2001).

Hasta el momento, los esfuerzos dirigidos hacia la evaluación y búsqueda de alternativas para el desarrollo en los sistemas de producción de esta comunidad han sido limitados y básicamente se han hecho desde un enfoque de investigación. La comercialización y mercadeo son deficientes ya que el agricultor no obtiene una ganancia justa por su producto, esto debido a que los canales de comercialización existentes no son los más adecuados, sumándose a ello el hecho de que la asistencia técnica no es oportuna ni pertinente ante la necesidad de los agricultores.

El presente trabajo tiene como propósito estudiar y resaltar la importancia de la gestión social y productiva de las actividades agrícolas que desarrolla el productor de la Comunidad de Yanallpa, con énfasis en el aspecto de la comercialización de su producción; además se pretende, a partir de la información que se obtenga del trabajo, sentar las bases para el inicio de procesos adecuados para la adopción de alternativas coherentes con las características sociales, económicas y gerenciales del productor local, que permitan mejorar la participación de los productores en el proceso de comercialización, lo que conllevaría a mejorar sus ingresos fortaleciendo su capacidad de negociación y haciendo frente a la falta de control sobre canales de comercialización por falta de capacitación y apoyo.

\section{MATERIAL Y MÉTODOS}

\section{Ubicación}

El estudio se realizó en la comunidad ribereña de Santa Cruz de Yanallpa, situada en la cuenca del Río Ucayali, tributario del Río Amazonas, en la jurisdicción del distrito de Jenaro Herrera, provincia de Requena y región Loreto. Dentro de las coordenadas geográficas $04^{\circ} 53^{\prime} 24^{\prime \prime}$ latitud sur y $73^{\circ} 46^{\prime} 47^{\prime \prime}$ longitud Oeste. Limita al Norte con el río Ucayali; al sur, con terrenos baldíos del Estado; al Este, con terrenos baldíos y al Oeste, con la comunidad 11 de agosto.

\section{Ecología}

Onern (1991), afirma que lquitos se considera ecológicamente como un bosque húmedo tropical; El clima de la zona de estudio se clasifica como húmedo y cálido, con una temperatura media anual de $26^{\circ} \mathrm{C}$ y una precipitación promedio anual de 2,600 mm. La estación invernal no es muy marcada y se caracteriza por un nivel de precipitación pluvial y temperatura ligeramente igual a la de las otras estaciones, además posee una elevada humedad relativa la cual fluctúa entre $80-88 \%$ según el SENAMHI.

\section{Caracterización biofísica}

La selva baja de la amazonía peruana abarca alrededor de 680000 km2 (Dourojeanni, 1990), en esta área, los ríos constituyen la más importante infraestructura, así como los llanos inundables significando más del $12 \%$ de la superficie total (Salo et al, 1986).

Existen tres grandes ríos en el área de Loreto; el Marañón que viene de la parte nor oriental, el Ucayali que nace en la parte sur del Perú y el Amazonas que tiene su origen en la confluencia de los dos anteriormente mencionados. Estos ríos son ricos en sedimentos, los cuales originan la formación de nuevas tierras dando lugar al establecimiento y la sucesión de la vegetación.

Así mismo Encarnación (1993), indica que los 
bosques son una manifestación de la diversidad biológica, presentando cierta similaridad en relación al clima, así para condiciones de suelos inundables los clasifica como Clase II, bosque bajial, sub clase A, diferenciables en dos grupos de bosques:

- Expuesto al flujo de agua blanca; tahuampas, pungales, arenales, barriales, restingas.

- Expuestas a aguas Negras.

En estos ecotonos destacan especies herbáceas y leñosas medianas como Heliconia marginata, $\mathrm{H}$. episcopalis, Ficus insípida, Maquira sp, Clarisia sp., Cecropia spp., Inga spp., Spondias mombin, Calycophyllum spruceanum, entre otras especies que se desarrollan en estos suelos de restinga.

El río Ucayali se extiende desde la confluencia del Tambo y el Urubamba hasta su desembocadura en el Amazonas. La cuenca alta es la correspondiente al departamento de Ucayali y la cuenca baja se ubica en el departamento de Loreto. Dos grandes paisajes marcan el territorio en la zona:

\section{Las tierras altas (tierra firme)}

Llamadas "altura" en la terminología local, libres de la inundación por el agua del río Ucayali. Esta unidad, ubicada de diez a más metros sobre el nivel de la llanura aluvial, está conformada por terrazas medias, altas y colinas de topografía plana, ondulada, leve - fuertemente disectada (topografía empinada). Los suelos, superficiales a moderadamente profundos, tienen origen en los sedimentos heterogéneos antiguos, depositados durante el terciario y el cuaternario. Debido a procesos de meteorización y lixiviación se han formado suelos arcillosos, francos y arenosos, muy ácidos, fuertemente carentes de bases, con una baja capacidad para el almacenamiento/retención de nutrientes y un nivel muy alto de aluminio intercambiable, tóxico para la mayoría de los cultivos agrícolas.

En las quebradas que drenan el agua de las lluvias de las tierras altas y las depresiones de las terrazas medias, el drenaje deficiente forma suelos hidrometamórficos moderadamente ácidos y de fertilidad natural media a baja.

El cambio constante del curso del cauce del río Ucayali modifica el aspecto de las riberas de las tierras altas, produciéndose una pérdida de terreno por erosión lateral originando barrancos de talud casi perpendicular.

Se aprecian tres tipos de biotopos de las tierras altas influenciados, principalmente, por el tipo de suelo el drenaje y la escorrentía; estos son:

- Terrazas medias: planicies, pendientes, caños y quebradas.

- Terrazas altas: planicies, pendientes y vallecitos de quebrada.

- Colinas: cimas, pendientes (de fuerte gradiente) y vallecitos de quebrada.

\section{Las tierras bajas (Varzea)}

Llamadas "bajo" en la terminología local, son inundadas anualmente por la creciente del río Ucayali. Dependiendo del caudal y la topografía del terreno la inundación puede alcanzar de $2 \mathrm{~m}$ a $3 \mathrm{~m}$ de profundidad y durar de 2 a 4 meses al año. Esta unidad está conformada por los complejos de orillares, terrazas bajas y la planicie aluvial, de topografía generalmente plana; con leve pendiente hacia los cuerpos de agua; ondulada o con elevaciones consecutivas (a modo de diques) y depresiones ("bajiales") entre estas.

Los suelos tienen origen en los sedimentos recientes y subrecientes acarreados por el río Ucayali y depositados durante el periodo de inundación. Después del retroceso del agua de inundación los suelos pueden quedar bien 0 imperfectamente drenados dependiendo del lugar y la topografía del terreno, de la distancia del río y de la permeabilidad del terreno.

En los suelos bien drenados de las islas estabilizadas, los complejos de orillares y las terrazas medias, el aporte continuo de sedimentos forma suelos con regular contenido de nutrientes, acidez baja, mayor capacidad de retención de nutrientes y un nivel bajo de toxicidad por aluminio. En los suelos pobremente drenados de las partes más planas y depresiones de las terrazas bajas y la planicie aluvial, el hidrometamorfismo origina suelos extremadamente ácidos con alto contenido de materia orgánica parcialmente descompuesta, en la capa superficial, y bajo contenido de nutrientes.

En la ribera de las tierras bajas, con una pendiente suave, el cauce meándrico del río forma nuevos suelos, "playas" y "barrizales", arena o limo respectivamente, depositados por el agua de inundación y que aparecen en la época de estiaje cuando las aguas de inundación se retiran.

Los diversos biotopos son influenciados principalmente por el tipo de sedimento (limo o arena), el tipo de agua de inundación (aguas blancas o aguas negras), la gradiente de inundación y el drenaje: Se pueden observar:

- Islas, playones y bancos de arena.

- Complejo de orillares: playas, barrizales, restingas bajas y bajiales.

- Terrazas bajas de drenaje moderado a bueno: restingas medias y altas.

- Terrazas bajas de drenaje muy pobre: bajiales. 
- Depresiones permanentemente inundadas: pantanos, aguajales, pungales.

\section{Tipo de investigación}

La evaluación se realizó con un enfoque de carácter participativo, basado en talleres de evaluación rural participativa; a través de entrevistas a productores de la comunidad de Yanallpa; así como, de la información documental generada por la operación del proyecto.

En la metodología que se empleó para ejecutar el presente estudio se ha considerado los aspectos de diseño de las encuestas así como la estructura y el tamaño de la muestra a utilizar. En esta fase también se consideró entrevistas no estructuradas y semi estructuradas.

\section{Población y muestra}

Para efectos del trabajo, se tomó como fuente de información a 10 familias asentadas en la comunidad y que representativamente fueron seleccionadas por su disponibilidad a cooperar con el presente trabajo de investigación, a las cuales se realizó las entrevistas y visitas de las parcelas. Esto para obtener una información detallada de las actividades económicas productivas.

\section{Procedimientos}

Se utilizaron instrumentos de recolección de información de tipo cuantitativo, cualitativo, talleres participativos, encuestas con entrevistas semi-estructuradas, análisis documental, observación de la producción llevando control de la producción y plática directa.

\section{Determinación del valor de la parcela tradicional}

Esta actividad se realizó en forma individual con cada agricultor, para ello se considero dos sub actividades:

Mediante el desarrollo de una encuesta estructurada. En la cual se evaluó el medio socio-cultural, para lo cual se consideró información como su organicidad familiar y local, sus actividades productivas, lugar de procedencia, conocimientos ancestrales, influencias por técnicas de manejo y aprovechamiento de los recursos naturales por efecto de la asistencia técnica externa.

Micro zonificación de las parcelas. Esta actividad se realizó con la finalidad de generar información sobre las potencialidades y limitaciones de las parcelas que a futuro sirva de base para la elaboración, aprobación y promoción de proyectos de desarrollo, planes de manejo en áreas y temas específicos en el ámbito local.
Igualmente, nos permitió conocer el ordenamiento y/o acondicionamiento de las parcelas, así como tener una idea del plan de producción que sigue los agricultores a través de la configuración de las parcelas.

\section{Inventarios y dispersión (frutales, forestales, hortícolas, etc.).}

Se realizó inventarios y dispersión de las Sp. frutales, Sp. forestales.

Para Sp. Frutales se realizó, marcándolo con Cinta Maskin Tape alrededor del tallo, pintando en el su número correspondiente con los marcadores finos, para su fácil conteo e identificación.

Para Sp. Forestales se realizó, marcándolo con Cinta Maskin Tape alrededor del tallo, pintando en el su número correspondiente con los marcadores finos, para su fácil conteo e identificación.

\section{Determinación del valor económico de la producción}

Para la determinación del valor económico de la producción, se evaluó los ingresos y los egresos que se generan en el proceso productivo por parte de los agricultores; así las actividades que se evaluaron fueron las siguientes:

\section{Para aquellos aspectos que generaron ingresos en las parcelas}

- Se cuantificó la producción de los principales cultivos.

- Se determinó los ingresos por cultivo y por parcelas.

- Se valoró la producción destinada al autoconsumo.

- Se determinó el ingreso total de la chacra por año.

\section{Para aquellos aspectos que generaron egresos en las parcelas}

- Se determinó los números de jornales utilizadas para las labores agrícolas por año.

- Se valoró el trabajo familiar, el trabajo solidario y el asalariado.

- Se evaluó los gastos en la compra de semillas y otros insumos.

- Se evaluó el gasto en el transporte dentro de las comunidades realizado en la post cosecha. 


\section{Determinación de la estructura agraria de la comunidad de Santa Cruz de Yanallpa}

Con el fin de poder estructurar la actividad agrícola en la comunidad se realizó encuestas semi estructuradas a cada uno de los agricultores, con la finalidad de concretizar las informaciones primarias y así poder caracterizar la actividad agraria de la comunidad; con el fin de poder homogenizar la información, en ciertos términos de referencia se incluyo dentro de las encuestas algunas características que nos permitieran tener una mejor percepción del tema como:

- Conocimiento de las prácticas agrícolas tradicionales.

- Mayor arraigo en la comunidad referido a procedencia local de la familia.

- Mayor disponibilidad para la intervención externa.

\section{Determinación de la organicidad para el trabajo agrícola en la comunidad de Yanallpa}

Este aspecto de mucha importancia se desarrollo evaluando el medio socio-cultural de la comunidad, para ello se considero su organicidad local, sus actividades productivas, lugar de procedencia de los miembros involucrados en el estudio, conocimientos ancestrales en el manejo de los cultivos, influencias por parte de instituciones externas en la transferencia de técnicas de manejo y aprovechamiento de los recursos naturales y de cómo ello influenció en su actividad agrícola y economía familiar.

\section{Métodos}

Se aplicó el diseño experimental ejecutándose sobre la base de los resultados de la encuesta y la descripción estadística de la muestra, para las estimaciones econométricas. Basada en una estadística del tipo cualitativa - cuantitativa, representanta en tablas de contingencia, tablas de distribución de frecuencia, medidas de tendencia central, gráficos.

Para el caso de tratamiento, en el presente trabajo de investigación se trabajó en forma particular con cada agricultor de la comunidad, que se seleccionó para efectos del trabajo, siendo los siguientes agricultores:

\section{Variables en estudio}

Variable independiente $(X)$

$$
\begin{array}{ll}
\text { X1 }= & \text { Capital } \\
\text { X2 } & \text { Tierra } \\
\text { X3 }= & \text { Mano de obra }
\end{array}
$$
X4 $=$ Comercialización.
X5 $=$ Diagnostico socioeconómico.
X6 $=\quad$ Sistemas de Cultivo

Variable Dependiente (Y)

$$
\begin{array}{ll}
\mathrm{Y} 1=\quad \begin{array}{l}
\text { Rentabilidad económica de las } \\
\text { huertas chacras. }
\end{array}
\end{array}
$$

\section{RESULTADOS}

\section{Determinación del valor de la parcela tradicional}

¿Qué precio de venta / alquiler da a su Huerto Chacra? La tabla 1 muestra la variable precio de las parcelas, las cuales fueron obtenidas de los propios agricultores de la zona, en ella se puede observar que el valor de la parcela mas que monetario es amical, ya que ella es parte de la familia, por lo que no existe un precio real de transacción.

Tabla 1: Precio de las parcelas

\begin{tabular}{lll}
\hline Respuesta & $\mathrm{fi}$ & $\%$ \\
\hline $\begin{array}{l}\text { 1. No tiene precio por ser una parcela } \\
\text { de mi familia. }\end{array}$ & 04 & 44,4 \\
$\begin{array}{l}\text { 2. Dependería de la oferta de la persona } \\
\text { que quiera comprar }\end{array}$ & 01 & 11,1 \\
$\begin{array}{l}\text { 3. No podría decirlo ya que el tiempo de } \\
\text { trabajo en mi chacra es de muchos } \\
\text { años. }\end{array}$ & 03 & 33,3 \\
$\begin{array}{l}\text { 4. Poseo muchas especies de plantas en } \\
\text { mi parcela no podría ponerle precio. }\end{array}$ & 01 & 11,1 \\
\hline Total & 09 & 100,00 \\
\hline
\end{tabular}

\section{La microzonificación de las parcelas y} el valor real de las chacras huerta

Para conocer lo que realmente posee cada agricultor al interior de su huerto chacra, se realizó la micro zonificación de las parcelas, en las cuales se caracterizó cada una de ellas, identificando las especies que poseen, lo que nos permitió entender su conocimiento local para el establecimiento de sus cultivos, la orientación productiva de los agricultores, como también de los cultivos de mayor capitalización dentro de las parcelas.

Las figuras 1, 2, 3 y 4 muestran las parcelas mas representativas encontradas en la comunidad de Yanallpa en ella se observa la forma de distribución de los cultivos y el uso del suelo de acuerdo a las características y necesidades de cada cultivo, lo cual hace de cada parcela un conocimiento propio de cada 
agricultor en manejar y entender su entorno, poniendo en práctica su comprensión ancestral en beneficio de su producción.

\section{Inventarios de las parcelas}

En la tabla 2, se observa las especies identificadas en las parcelas de los agricultores, ella se observa las parcelas 2, 3, 8 y 9 que son las más representativas por su alta diversidad de especies y su forma especial de distribución.
La tabla 3, nos muestra la forma de utilización del suelo por parte de los agricultores, en ella observamos como el agricultor destina cada parte de su parcela a una actividad específica, para ello toma en cuenta las condiciones del suelo con el fin de que sus cultivos no se vean afectados a futuro, esto es algo muy importante porque este conocimiento tradicional está relacionado con las características del cultivo es decir a su necesidad de Luz, nutrientes, estacionalidad, inundaciones, etc., donde la utilización de las zonas inundables son hoy las mas utilizadas por el impulso

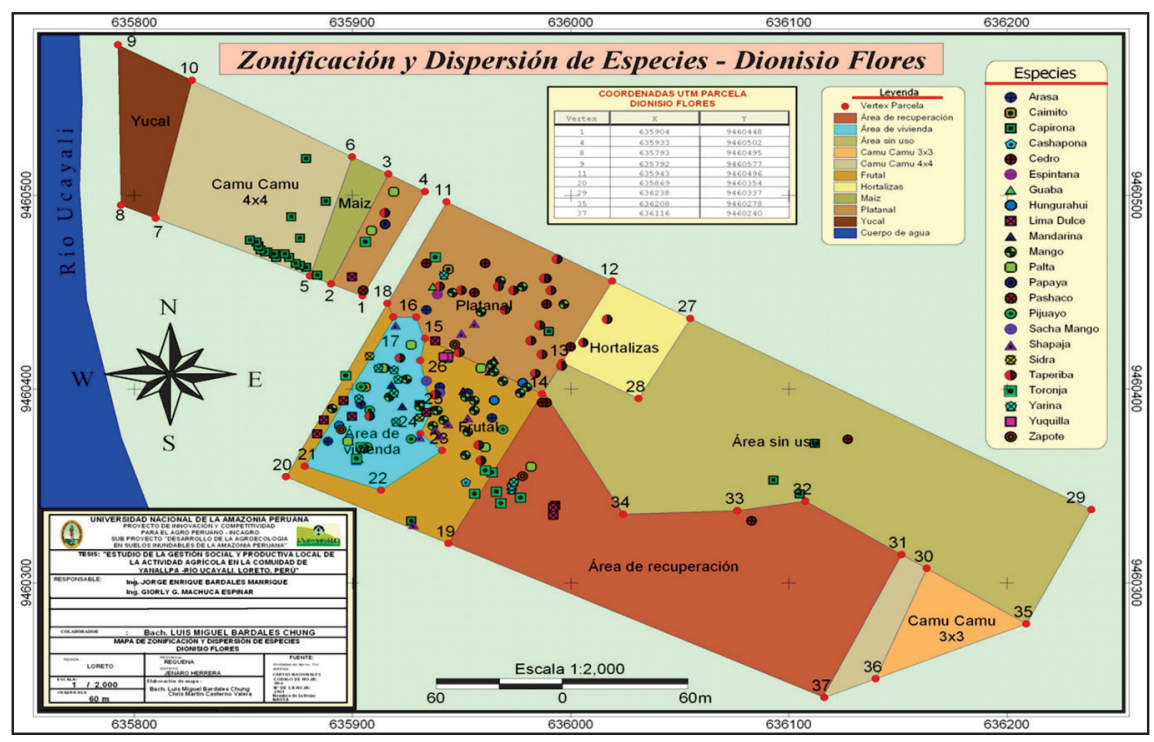

Figura 1: Zonificacion y Dispersion de especies Parcela 02.

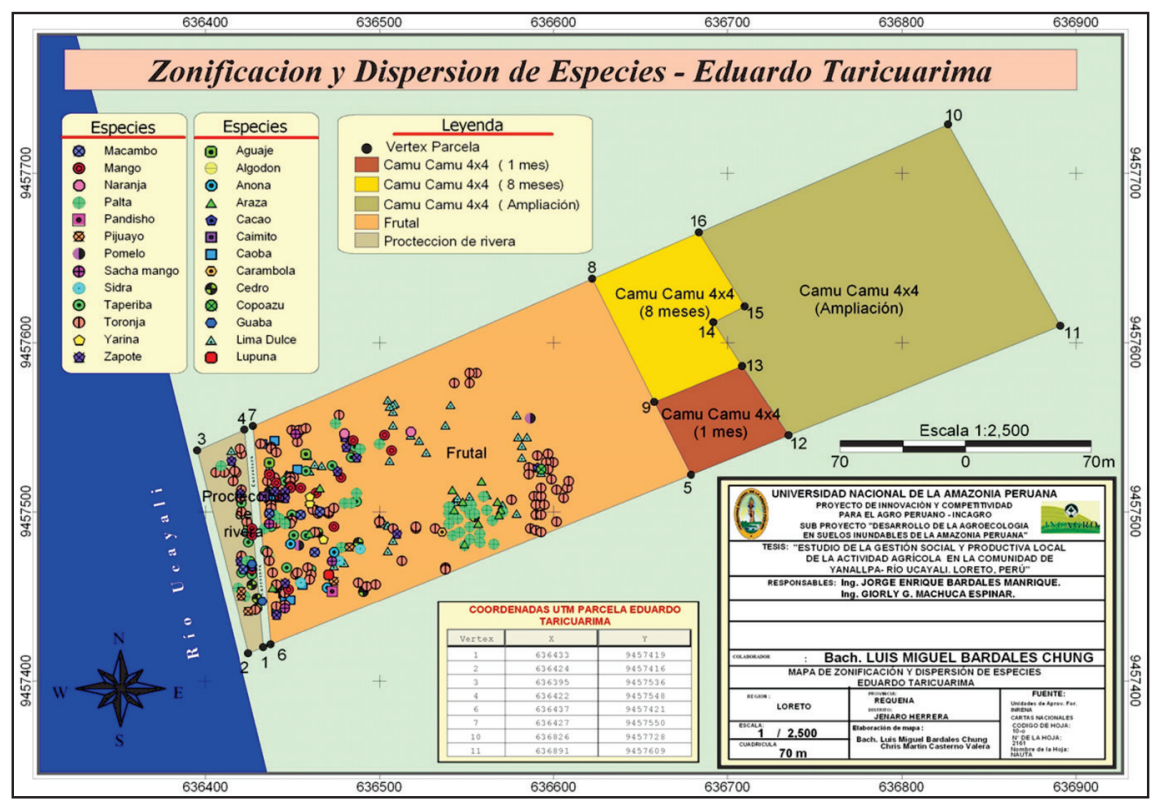

Figura 2: Zonificacion y Dispersion de especies Parcela 03. 


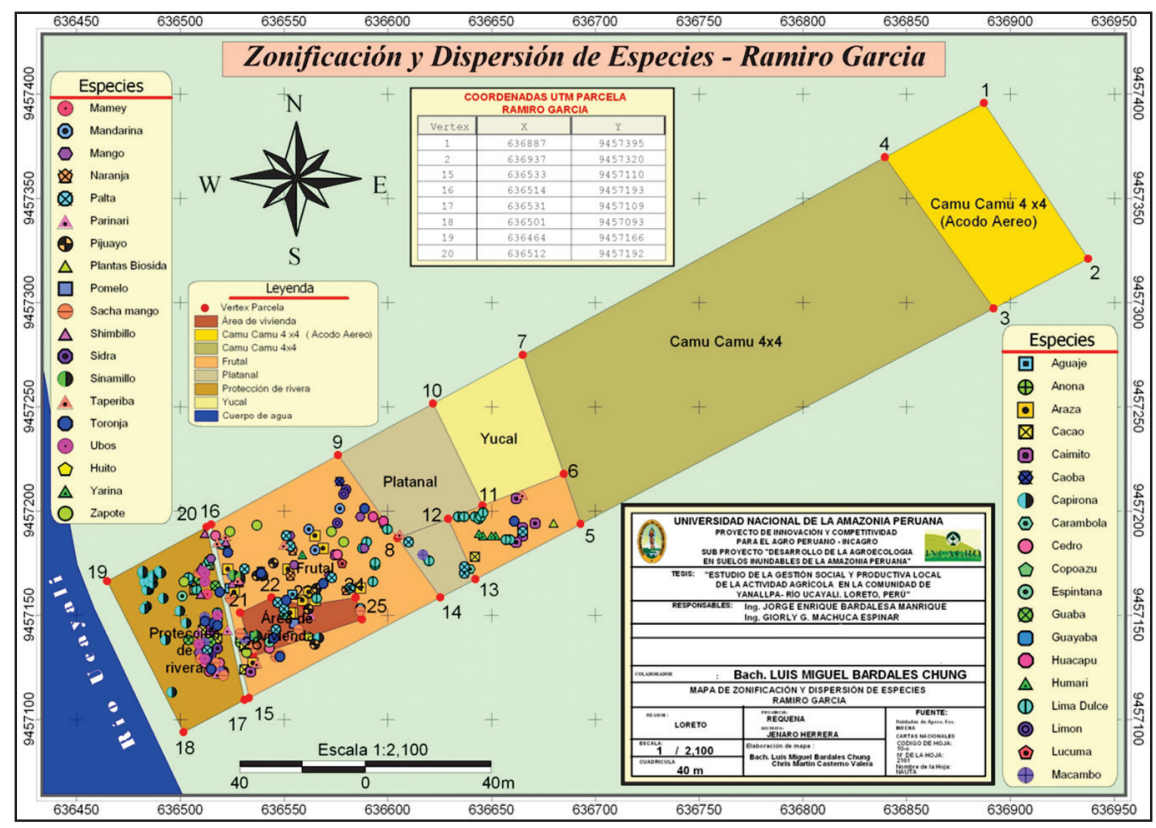

Figura 3: Zonificacion y dispersion de especies parcela 08.

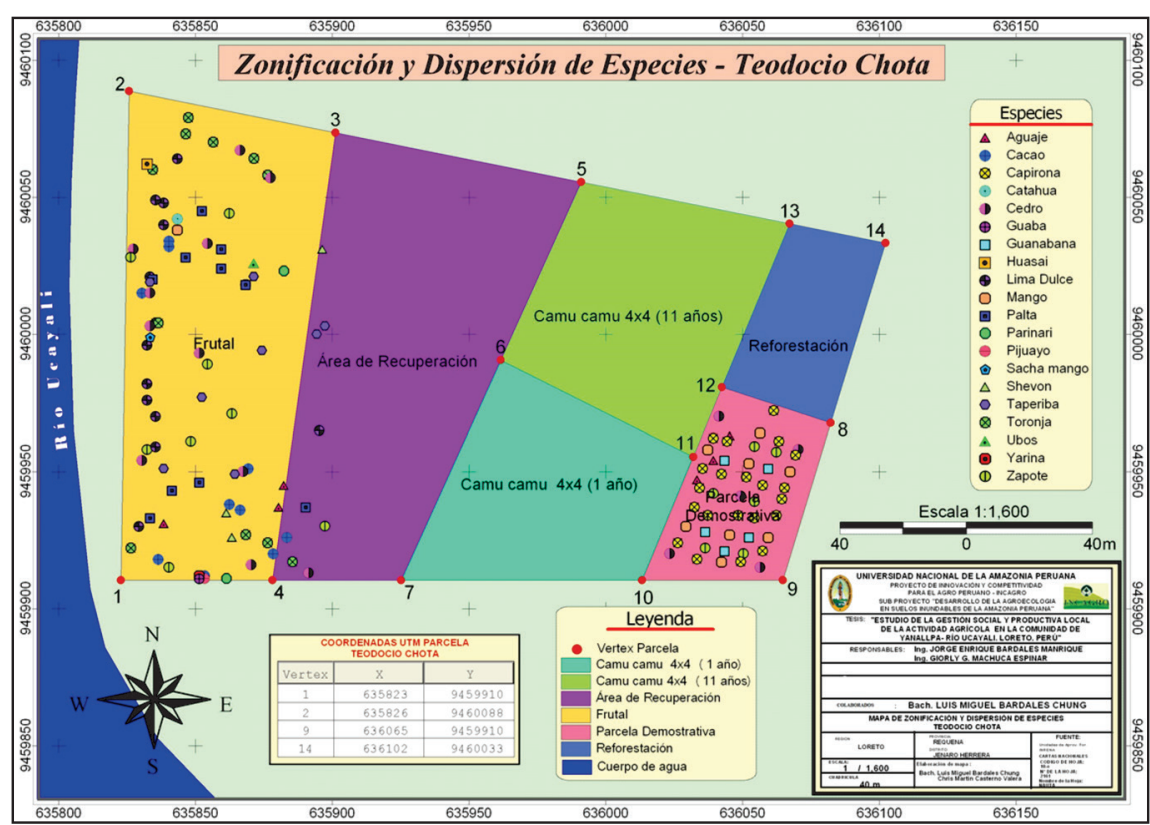

Figura 4: Zonificacion y dispersion de especies parcela 09.

en la explotación del Camu Camu.

\section{Análisis de datos}

Con los datos obtenidos del inventario de caracterización de los huertos chacras, se realizó el análisis de los datos, utilizándose índices de diversidad y heterogeneidad, así como un coeficiente de similaridad.

a. Índices de Margalef y Shannon
La tabla 4, nos muestra el análisis de datos para las parcelas en estudio, donde se puede observar que la parcela 2, muestra la mayor diversidad de especies con respecto a las demás parcelas y con cierto grado de heterogeneidad, así mismo presenta el segundo lugar en numero de especies y cuarto lugar en número de individuos, en comparación a la parcela 03 que posee el segundo lugar en diversidad, pero presenta una baja heterogeneidad, ocupa el segundo lugar en riqueza de especies y primer lugar en número de individuos. 
Tabla 2: Especies en las parcelas.

\begin{tabular}{|c|c|c|c|c|}
\hline Inventario & $\begin{array}{c}\text { Parcela } \\
02\end{array}$ & $\begin{array}{c}\text { Parcela } \\
03\end{array}$ & $\begin{array}{c}\text { Parcela } \\
08\end{array}$ & $\begin{array}{c}\text { Parcela } \\
09\end{array}$ \\
\hline Aguaje & & 2 & 7 & 6 \\
\hline Anona & & 3 & 1 & \\
\hline Algodón & & 1 & & \\
\hline Arazá & 8 & 13 & 15 & \\
\hline Cacao & & 1 & 10 & \\
\hline Caimito & 2 & 1 & 8 & \\
\hline Caoba & & 11 & 3 & \\
\hline Cacao & & & & 10 \\
\hline Capirona & 24 & & 20 & 21 \\
\hline Cashapona & 1 & & & \\
\hline Carambola & & 1 & 2 & \\
\hline Cedro & & 11 & 11 & 17 \\
\hline Catahua & & & & 1 \\
\hline Copoazu & & 1 & 1 & \\
\hline Espintana & 1 & & 3 & \\
\hline Guaba & 2 & & 4 & 1 \\
\hline Guanabana & & & & 5 \\
\hline Guayaba & & & 1 & \\
\hline Huacapu & & & 2 & \\
\hline Huito & & & 1 & \\
\hline Hungurahui & 3 & & & \\
\hline Huasai & & & & 1 \\
\hline Humari & & & 2 & \\
\hline Lima dulce & 11 & 44 & 21 & 12 \\
\hline Limón & & & 4 & \\
\hline Lupuna & & 1 & & \\
\hline Lúcuma & & & 2 & \\
\hline Macambo & & 5 & 6 & \\
\hline Mamey & & & 3 & \\
\hline Mandarina & 5 & & 3 & \\
\hline Mango & 23 & 16 & 8 & 9 \\
\hline Naranja & & 2 & 3 & \\
\hline Palta & 9 & 36 & 16 & 10 \\
\hline Parinari & & & 4 & 2 \\
\hline Pan del Árbol & & 1 & & \\
\hline Papaya & 1 & & & \\
\hline Pashaco & 1 & & & \\
\hline Pijuayo & 5 & 5 & 6 & 1 \\
\hline Camu camu & 671 & 1868 & 1440 & 734 \\
\hline Pomelo & & 2 & 1 & \\
\hline Sacha mango & 2 & 4 & 12 & 1 \\
\hline Shapaja & 11 & & & \\
\hline Shimbillo & & & 5 & \\
\hline Sidra & 2 & 3 & 1 & \\
\hline Shevon & & & & 3 \\
\hline Sinamillo & & & 3 & \\
\hline Taperiba & 23 & 18 & 7 & 8 \\
\hline Toronja & 11 & 95 & 14 & 13 \\
\hline Ubos & & & 6 & 1 \\
\hline Yarina & 11 & 3 & 5 & 1 \\
\hline Sapote & 3 & 15 & 6 & 14 \\
\hline Total & 52 & 90 & 116 & 74 \\
\hline
\end{tabular}

Tabla 3: Áreas definidas en las parcelas.

\begin{tabular}{|c|c|c|c|c|}
\hline Inventario & $\begin{array}{c}\text { Parcela } \\
02\end{array}$ & $\begin{array}{c}\text { Parcela } \\
03\end{array}$ & $\begin{array}{c}\text { Parcela } \\
08\end{array}$ & $\begin{array}{c}\text { Parcela } \\
09\end{array}$ \\
\hline Área de recuperación & 1,354 & & & 1,094 \\
\hline Área de vivienda & 0,300 & & 0,098 & \\
\hline Área sin uso & 1,644 & & & \\
\hline Camu Camu $3 \times 3$ & 0,163 & & & \\
\hline Camu Camu $4 \times 4$ & 0,655 & 2,988 & 2,305 & 1,174 \\
\hline Frutal & 0,466 & 2,981 & 0,885 & 1,126 \\
\hline Hortalizas & 0,192 & & & \\
\hline Maíz & 0,092 & & & \\
\hline Platanal & 0,666 & & 0,319 & \\
\hline Protección de Rivera & & 0,240 & 0,357 & 0,257 \\
\hline Yucal & 0,201 & & 0,261 & 0,306 \\
\hline Total general & 5,733 & 6,209 & 4,225 & 3,957 \\
\hline
\end{tabular}

b. Coeficiente de similitud de Sorensen.

La matriz de similaridad (tabla 5) muestra que los huerto chacras 2 (Sr. Dionisio flores) y el 8 (Sr. Ramiro García), son los más similares, con un $51 \%$ de especies compartidas. Los huertos chacras que presentan menos similaridad por el menor número de especies compartidas son el 1 y 8 con $5 \%$ de especies compartidas.

\section{Determinación del valor económico de la producción}

Para el desarrollo de la presente variable, se identifico entre los agricultores los cultivos de mayor capitalización en su parcela, es decir aquellos cultivos que le generan ingresos en su mercado local interno y externo. Para lo cual se ha elaborado la tabla 6, en la que se muestra por agricultor los cultivos de mayor importancia en su parcela.

\section{Aspectos que generaron ingresos en las parcelas}

Ingreso total de la chacra por año/campaña.

Con los valores obtenidos de la valoración de la producción por cultivo, se elaboro la tabla 7 , en la cual se puede observar el ingreso por parcela por campaña y una aproximación de lo que se podría estimar el ingreso mensual de cada agricultor producto de los ingresos obtenidos de su producción.

En la tabla 7, se puede observar los ingresos que cada agricultor obtiene por campaña, esto es muy importante ya que a partir de ello se puede conocer la capacidad de inversión que el agricultor posee para poder reinvertir en su parcela, es decir en nuevos in put, así mismo la capacidad del agricultor de poder invertir en tecnologías que tiendan a mejorar la producción en la parcela. 
Tabla 4: Índices de Margalef y Shannon en los huerto-chacra estudiados.

\begin{tabular}{lrrrrrrrrr}
\hline Inventario & 01 & \multicolumn{1}{c}{02} & 03 & 04 & 05 & 06 & 07 & 08 & 09 \\
\hline Riqueza de especies (S) & 04 & 24 & 27 & 10 & 12 & 03 & 08 & 39 & 21 \\
Nro. de individuos (N) & 297 & 841 & 2165 & 136 & 457 & 183 & 294 & 1669 & 871 \\
Índice de Margalef & 0,52 & 3,43 & 3,39 & 2,14 & 1,80 & 0,38 & 1,23 & 5,12 & 2,95 \\
Índice de Shannon & 0,14 & 1,03 & 0,73 & 1,54 & 0,63 & 0,09 & 0,20 & 0,86 & 0,83 \\
\hline
\end{tabular}

Tabla 5: Matriz de similaridad del huerto-chacra estudiado.

\begin{tabular}{llllllllll}
\hline & 01 & 02 & 03 & 04 & 05 & 06 & 07 & 08 & 09 \\
\hline 01 & & & & & & & & & \\
02 & 0.29 & & & & & & & & \\
03 & 0.06 & 0.51 & & & & & & & \\
04 & 0.42 & 0.41 & 0.27 & & & & & & \\
05 & 0.13 & 0.22 & 0.15 & 0.36 & & & & & \\
06 & 0.29 & 0.15 & 0.07 & 0.31 & 0.07 & & & & \\
07 & 0.33 & 0.44 & 0.17 & 0.44 & 0.20 & 0.36 & & & \\
08 & 0.05 & 0.51 & 0.70 & 0.29 & 0.20 & 0.10 & 0.21 & & \\
09 & 0.24 & 0.49 & 0.46 & 0.52 & 0.18 & 0.17 & 0.28 & 0.43 & \\
\hline
\end{tabular}

Tabla 6: cultivos de mayor importancia por agricultor.

\begin{tabular}{|c|c|c|c|c|c|c|c|c|c|c|}
\hline Especie & $\begin{array}{c}\text { Parcl } \\
01\end{array}$ & $\begin{array}{c}\text { Parcl } \\
02\end{array}$ & $\begin{array}{c}\text { Parcl } \\
03\end{array}$ & $\begin{array}{c}\text { Parcl } \\
04\end{array}$ & $\begin{array}{l}\text { Parcl } \\
05\end{array}$ & $\begin{array}{l}\text { Parcl } \\
06\end{array}$ & $\begin{array}{c}\text { Parcl } \\
08\end{array}$ & $\begin{array}{l}\text { Parcl } \\
09\end{array}$ & $\begin{array}{c}\text { Parcl } \\
07\end{array}$ & $\begin{array}{l}\text { Total } \\
\text { general }\end{array}$ \\
\hline Aguaje & & & & & & & 7 & 6 & & 13 \\
\hline Arazá & & 8 & 13 & & & & 15 & & & 36 \\
\hline Cacao & & & & & & & 10 & 10 & & 20 \\
\hline Caimito & & & & & & & 8 & & & 8 \\
\hline Capirona & & & & & & & & & 2 & 2 \\
\hline Guanabana & & & & & 6 & & & 5 & & 11 \\
\hline Lima Dulce & 4 & 11 & 44 & 34 & & & 21 & 12 & & 126 \\
\hline Macambo & & & & & & & 6 & & & 6 \\
\hline Mango & & 23 & 16 & 7 & & & 8 & 9 & & 63 \\
\hline Palta & & 9 & 36 & & & & 16 & 10 & & 71 \\
\hline Pijuayo & & & & & & & 6 & & & 6 \\
\hline Sacha mango & & & & & & & 12 & & & 12 \\
\hline Taperiba & & 23 & 18 & & & & 7 & 8 & 1 & 57 \\
\hline Toronja & & 11 & 95 & & & & 14 & 13 & & 133 \\
\hline Ubos & & & & & & & 6 & & & 6 \\
\hline Yarina & & 11 & & & & & & & & 11 \\
\hline Plátano & & 833 & & 390 & 223 & & 798 & & 705 & 2949 \\
\hline Yuca & & 502 & & 650 & & & 1,346 & & & 2498 \\
\hline Maíz & & 4600 & & & & & & & & 4600 \\
\hline camu camu & 289 & 671 & 1,868 & 57 & 388 & 284 & 1,440 & 734 & 180 & 5911 \\
\hline Sapote & & 3 & 15 & & & & 6 & 14 & & 38 \\
\hline Total general & 293 & 6702 & 2105 & 1138 & 617 & 284 & 3,726 & 821 & 888 & 16,574 \\
\hline
\end{tabular}

\section{Aspectos que generan egresos en las parcela}

Las actividades que los agricultores desarrollan estan en funciones muchas veces a las actividades que ellos tiene dentro de su comunidad, asi la agricultura, la pesca y la caza son actividades con niveles de prioridades que ellos dan; asi por ejemplo, el de recolectar maderaje nuevo para renovar la casa, muchas veces complementa con actividades de caza, apertura de nuevas chacras, etc. Todas estas actvidades le generan egresos antes que ingresos, que son solventados con la actvidad agricola, por ello la agricultura es un medio que se podria como una inversión a futuro, ya que ella va ha habilitar al agricultor y le va a permitir poder mejorar su calidad de vida con los ingresos por 
Tabla 7: Consolidado de Ingreso por Parcela/Campaña.

\begin{tabular}{clcc}
\hline Parcela No & \multicolumn{1}{c}{ Agricultor } & $\begin{array}{c}\text { Ingreso por } \\
\text { campaña (01 año) }\end{array}$ & $\begin{array}{c}\text { Ingreso } \\
\text { mensualizado }\end{array}$ \\
\hline 02 & Luis Dionisio Flores Ampuero & 7604,00 & 633,67 \\
08 & Francisco Ramiro García Ahuanari & 6335,00 & 527,91 \\
03 & Eduardo Taricuarima Yarahua & 2739,00 & 228,25 \\
09 & Teodosio Chota Alvarado & 4841,00 & 403,42 \\
\hline
\end{tabular}

la venta de sus excendentes.

Asi las actividades que le generan egresos estarian dados de la siguiente manera:

- Actividad agricola.

- Caza y Pesca.

- Infraestructura.

- Compromisos familiares

- Actvidades ceremoniales.

\section{Organicidad para el trabajo agrícola en la Comunidad de Yanallpa}

La familia es el núcleo central de la actividad agrícola en la comunidad de Yanallpa, ya que de ella depende el cuidado de la chacra y las diversas actividades que se desarrollan como la siembra, cosecha, manejo de las plantas tiernas, etc.

Dentro del grupo de los agricultores en los cuales se encuentra inmerso el trabajo de investigación, también esta el grupo de personas que cooperan en eventos productivos de distintas denominaciones ("minga", "corta-mañana", "rueda", "corta tarde"), lo hacen en función a ciertas actividades, según cultivo, epoca de año, implementación de nuevas parcelas, asi como ciertas afinidades personales que se manifiestan también en otros aspectos de la convivencia social como son los lazos de parentesco y de alianza matrimonial, la corresidencia o vecindad. Los grupos de personas que se agrupan bajo este aspecto y suelen cooperar entre ellas con cierta regularidad son denominados como grupos de solidaridad laboral.
Las otras formas de asociación que se presentan en la comunidad son aquellas que suelen compartir alimentos y bienes, a estos se denominan grupos de solidaridad distributiva, y aquellos que suelen unierse con el fin de concelebrar eventos festivos, se los denomina grupos de solidaridad ceremonial.

Estos grupos tienen en mente el principio de la reciprocidad (ayuda mutua) que actúa en su armazón moral como un derecho y una obligación (Gasché, 2001).

En base a lo observado y tomando como base lo descrito por Oré (2006), se toma el diagrama que resume acertadamente la organización para el trabajo en las comunidad de yanallpa, tal como se muestra en la (figura 5).

\section{DISCUSIÓN}

Los huerto-chacras estudiados en los agricultores involucrados dentro del proyecto, se caracterizan por su alta diversidad de especies frutales, alimenticias, forestales, entre otras., siendo mucho mayor el número de especies frutales comparado con las otras especies encontradas; esta condición también es reportada por otros estudios llevados a cabo en la misma zona, tales como Oré (2006); De Jong (1995).

Los resultados encontrados reportan diferencia entre el número de especies presentes entre los huertochacras evaluados en los agricultores, esto debido probablemente a varios factores como disponibilidad

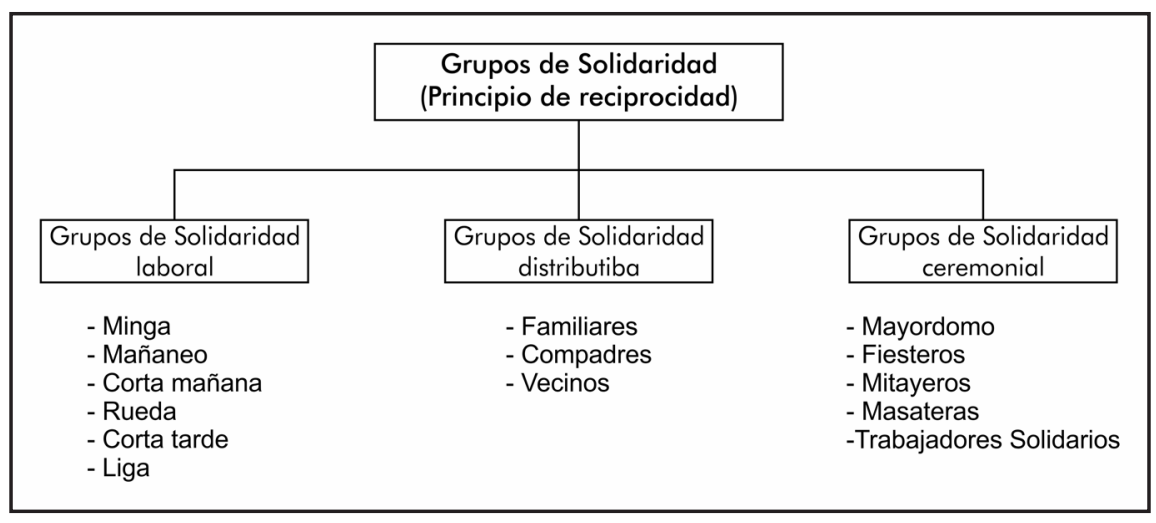

Figura 5: Diagrama de los Grupos de Solaridad en Yanallpa. 
de terreno, a las condiciones biofísicas propias de cada chacra y al tiempo de residencia de la familia propietaria del lugar en la zona, lo que en muchos casos va a determinar una mayor diversidad de especies en las parcelas; quienes realizaron un muestreo de huertas en la comunidad ribereña de Santa Rosa, bajo río Ucayali, muestran la diversidad de especies presentes así como la gran variabilidad en área y composición. También describen la evolución de las huertas en el pueblo y concluyen que es necesaria mayor investigación científica sobre las huertas amazónicas.

La identificación de especies de familias Botánicas de mayor importancia para los agricultores, caso familia de las Rutáceas (Cítricos) de mayor predominancia en los huerto-chacras estudiados reflejan la importancia que tiene para la población local el cultivo de plantas que puedan satisfacer necesidades básicas, entre otras especies de importancia medicinal, alimenticio y material de construcción, pero además el cuidado del suelo; Esto es corroborado en los trabajos realizados por Oré et al (1999), Padoch \& De Jong (1991), De Jong (1995); en la misma área del presente estudio, y Fernández et al (1983) en Tanzania; donde la mayoría de las plantas cultivadas en las comunidades estudiadas fueron sustraídas del bosque durante un largo proceso de domesticación y adaptación.

Para explicar esto De Jong (1995); mediante su trabajo de caracterización de los huertos-chacra o jardines de casa de restinga de la comunidad de Yanallpa, destacando la incorporación de especies de árboles nativos de selva baja dentro de los terrenos de cultivo de los agricultores locales. Considera los huertoschacra como formas de agricultura altamente variable, más que cualquier otra forma descrita antes.

La actividad de caza y pesca, para estos agricultores no es una prioridad esto nos demuestra sus acciones desarrolladas por los agricultores con el fin de obtener fuente de proteína para la alimentación, y a pesar de ello le genera egresos antes que ingresos, ya que el agricultor debe contar con los materiales necesarios para la pesca, los cuales a pesar de ser gastos mínimos no son renovados continuamente, aquí se resalta que los excedentes de la pesca muchas veces no son negociados si no mas bien son distribuidos entre los miembros de la familia, de igual forma la caza esta orientada a la obtención de proteína animal y va acompañada muchas veces de otras actividades que realiza el agricultor local. Limachi y Farroñay (1999), en trabajos evaluados determinaron, que aunque existen casos de familias más dedicadas a la agricultura y otras actividades, los pobladores distribuyen su tiempo en todas estas y esta diversificación obedece a una estrategia de minimización de riesgos y generación de un ingreso permanente y estable durante todos los meses del año; caracterizado por una alta variabilidad de precios para sus productores y un régimen hidrológico inestable.

Los compromisos familiares y sociales, son de gran responsabilidad para el agricultor, ya que la educación de los hijos es algo primordial en ellos, esto le genera mayor dedicación a sus chacras, a pensar en ampliarla, con el fin de poder cumplir con los compromisos familiares, este sentimiento de superación familiar está permanente en los agricultores de la Comunidad, ya que constantemente ellos reciben capacitaciones no sólo con conocimientos teóricos y prácticos, sino participan en diferentes eventos donde ganan experiencias en gestión y manejo administrativo de sus chacras huertas y es por ello que le dan un nuevo enfoque a los compromisos con los integrantes de su núcleo familiar.

De igual manera, las actividades ceremoniales, son de gran importancia en cada comunidad, como las fiestas patronales, el aniversario y otros eventos relacionados a la protección de la diversidad cultural y en la cual cada poblador de la comunidad de Yanallpa, tiene una tarea especifica ya sea en ayuda monetaria, en viveres o en apoyo administrativo de gestión, en la cual el agricultor destina sus recursos para poder cumplir con este fin en la cual la comunidad socializa su aniversario invitando a otras comunidades en la cual ellos se comprometen con todo los gastos para sus invitados inclusive para los que llegen a la comunidad durante sus fiestas siendo o no invitados. Aquí interviene mucho la palabra comprometida de los grupos de solidaridad ceremonial, acción que demuestra la relevancia de los compromisos asumidos por los pobladores en las distintas fiestas patronales de su comunidad.

Al respecto, existen nuevas corrientes filosóficas en las cuales se toma en cuenta las perspectivas propias de los campesinos, respecto a su cosmovisión y su forma de vida; una de ella es la "coevolución integral entre conocimiento y desarrollo" (Norgaard \& Sikor, 2001). Una de las características más importantes de la perspectiva coevolucionista es que otorga legitimidad al conocimiento cultural y experimental de los agricultores. Defiende el concepto de que sus formas de razonamiento pueden no traducirse como formas de razonamiento científico, pero el "cómo y qué" entendido por ellos ha probado ser apto para su sistema y puede usarse para comprender ese sistema.

En cuanto al control de plagas en sus cultivos, está orientada en Prácticas Culturales, Manejo del Cultivo, Uso de plantas Biocidas, Conocimientos tradicionales, actividades que muestran las acciones que realizan los productores de la Comunidad que se consideran como prácticas de manejo y son propias de los conocimientos ancestrales, que ha ido pasando de generación en generación y que nos dan referencia 
que existe relación y coherencia con los conocimientos que en la agricultura convencional son utilizadas dentro del MIP como parte del control Cultural. Este hecho, es muy importante, ya que corroboramos una vez más que es necesario e importante el acceso a la Cultura local, protección y promoción de la diversidad cultural, donde se aplique estrategias para armonizar el conocimiento académico con los conocimientos tradicionales de las comunidades.

Así el calendario agrícola, es desarrollado en base a la época en que el cultivo produce y de las fases de producción de los cultivos de los agricultores, observándose que los huertos chacras poseen un sistema de producción durante todo el año, permitiéndoles poder asumir compromisos familiares, ceremoniales, etc. Esto demuestra que existe cierta sostenibilidad agrícola, donde cada agricultor busca tener mayor producción cada campaña y satisfacer las necesidades de su núcleo familiar, a pesar de la existencia de cambios que se puedan presentar, durante el proceso de producción y post cosecha de los cultivos trabajados en la campaña. Además, los resultados reportan una gran diferencia entre el número de especies presentes entre los huertochacras evaluados, esto debido probablemente a la disponibilidad de terreno, a las condiciones biofísicas propias de cada caso y al tiempo de residencia de la familia propietaria del lugar; esto es mencionado también por Piland (2000) en un estudio llevado a cabo en el pueblo Tsimane de Bolivia.

Algunas conclusiones son:

El valor de sus chacras para los agricultores, más que económico como un bien de transacción, es un valor de posesión ya que los agricultores y sus familias tiene una identificación muy grande hacia sus tierras y las ven como un sustento para toda la vida de sus generaciones futuras, tal y como se muestra en la tabla 3 , donde la propiedad familiar es muy significativa, así lo representan el $44,4 \%$ de los agricultores.

Al analizar las especies dentro de la parcela, determinamos que el agricultor tiene cultivos que se denominan de capitalización ya que durante el año le permite obtener ingresos y poder cumplir con sus compromisos familiares y sociales; se observa que cultivos como la lima dulce, la taperiba y palta son los cultivos que mayor ingreso generan.

Es importante saber que la principal fuente de ingreso en la comunidad es la agricultura, en ella se basa el mayor esfuerzo de los agricultores tanto para el aprovechamiento de sus parcelas, la comercialización de sus frutas, etc., quedando en segundo plano la pesca y la caza; esto se ve reflejado en el destino de la producción donde el $70 \%$ de los agricultores manifiesta que está destinada al consumo y el $30 \%$ a la venta, así mismo a la calidad del gasto que las familias efectúan, que está más orientado a su parcela, y a la adquisición de herramientas que faciliten el trabajo de campo y le permitan maximizar su esfuerzo en la busca de generar mayores ingresos.

La microzonificación de los huertos chacras, nos permitió conocer la estructura de los mismos, de cómo fueron concebidos por los agricultores mediante la aplicación de sus conocimientos tradicionales, la dispersión en la parcela, densidades de siembra que están muy relacionadas con la fertilidad natural de los suelos, el uso de las áreas de la parcela en las que se implementan los cultivos tomando en cuenta el hábitat de cada especie. Un ejemplo de ello es que los agricultores en su mayoría destinan las restingas bajas para el cultivo de camu camu, cultivo que representa una expectativa muy grande para el futuro de sus familias.

El inventario de los huertos chacras determinó que existe una alta diversidad de especies vegetales presentes en ellas, lo cual representa la armonía de las relaciones entre los agricultores y su medio ambiente, pues en ellas puede encontrarse plantas silvestres y espontáneas que aún sin tener valor utilitario o comercial, son cuidadas y protegidas con igual esmero y con prácticas agrícolas limpias.

Las parcelas con mayor diversidad de especies, distribución y uso de las áreas, son la parcela 2 con 22 especies, pertenecientes a 19 géneros y a 14 familias botánicas, con un total de 841 individuos, con un área de 5,77 Has; la parcela 3 con 26 especies, pertenecientes a 19 géneros y a 174 familias botánicas, con un total de 2165 individuos y con un área de 6,209 Has, la parcela 8 con 36 especies, pertenecientes a 26 géneros y a 16 familias botánicas, con un total de 1669 individuos y un área de 4,225 Has y la parcela 9 con 22 especies, pertenecientes a 19 géneros y a 14 familias botánicas, con un total de 871 individuos y un área de 3,957 Has.

Los índices de diversidad encontrados en los huertochacras están determinados en parte por factores medioambientales, pero principalmente por las necesidades e intereses de sus propietarios, la parcela 2 muestra la mayor diversidad de especies con respecto a las demás parcelas y con cierto grado de heterogeneidad, en comparación a la parcela 3 que presenta el segundo lugar en diversidad. Así la matriz de similaridad muestra que el huerto chacra 2 y el 8, son muy similares con un $51 \%$ en el número de especies compartidas, en comparación a las huertos chacras 1 con respecto al huerto 8 con un solo $5 \%$ de similitud en especies compartidas.

Los huerto-chacras representan un valioso banco genético y una forma interesante de conservación in situ de cultivos nativos de la Amazonía peruana. 
Los saberes locales y el trabajo solidario siguen estando vigentes en la población de Yanallpa, no obstante la intervención de agentes de desarrollo que promueven la modernización agrícola, por lo general, tendientes a la homogenización de chacras con cultivos comerciales, Desconocer la cosmovisión local y peor aún, no comprenderla a pesar de conocerla; puede poner en riesgo el éxito de los proyectos de desarrollo en comunidades ribereñas como Yanallpa.

\section{REFERENCIAS \\ BIBLIOGRÁFICAS}

De Jong, W. (1995). Diversity, Variation, and Change in Ribereño Agriculture and Agroforestry. Wageningen Holanda: Landbouwuniversiteit.

Dourojeanni, R. (1990). Amazonía.- qué hacer? Centro de estudios teológicos de la Amazonía, lquitos (Perú). lquitos (Perú).

Encarnación, F. (1993). El bosque y las formaciones vegetales en la llanura amazónica del Perú. UNMSM. Alma Mater, 6, 95-114.
Gasché , J. (2001). Biodiversidad domesticada y manejo hortico-forestal en pueblos indígenas de la Amazonía. Agroforestería en las Américas, 8(32), 28-34.

Leff, E. (26-28 de noviembre de 2001). Agroecología y Saber Ambiental . Porto Alegre.

Onern. (1991). Estudio detallado de suelos y reconocimiento de cobertura y uso de la tierra. (lquitos) Informes, anexos y mapas.

Oré , I., Delgado , O., Llapapasca , C., Kvist , L., \& Gonzales, A. (1999). Composición, similaridad y uso de especies cultivadas en huertas domésticas de la Reserva Nacional Pacaya - Samiria. Revista Conocimiento, 5((2)), 141-157.

Oré, I. (2006). Agrodiversidad y Saberes en el Huertochacra Amazónico. Caso de la Comunidad de Yanallpa, Río Ucayali, Loreto, Perú. . Tesis para optar título de Maestro en Ciencias, UNAS, Amazonia .

Salo, J., Kalliolla, R., Hakkinen, I., Makinen , Y., Niemela, P., Puhakka, M., \& Coley, P. D. (1986). River dynamics and the diversity of Amazon lowland forest. Nature, 322, 254-258. 